\title{
Laser and Optical System for Laser Assisted Hydrogen Ion Beam Stripping at SNS
}

\section{Corresponding Author:}

\section{Yun Liu}

15 Spallation Neutron Source

16 Oak Ridge National Laboratory

171 Bethel Valley Road, Oak Ridge, TN 37831 USA

18 Phone: (865) 241-2063

19 Email: liuy2@ornl.gov

21 * Notice of Copyright: This manuscript has been authored by UT-Battelle, LLC under Contract No. 22 DE-AC05-00OR22725 with the U.S. Department of Energy. The United States Government retains 23 and the publisher, by accepting the article for publication, acknowledges that the United States 24 Government retains a non-exclusive, paid-up, irrevocable, world-wide license to publish or reproduce 25 the published form of this manuscript, or allow others to do so, for United States Government 26 purposes. The Department of Energy will provide public access to these results of federally sponsored 27 research in accordance with the DOE Public Access Plan (http://energy.gov/downloads/doe-public28 access-plan). 
3 Recently, a high-efficiency laser assisted hydrogen ion (H-) beam stripping was 4 successfully carried out in the Spallation Neutron Source (SNS) accelerator. The

5 experiment was not only an important step toward foil-less $\mathrm{H}$ - stripping for charge 6 exchange injection, it also set up a first example of using megawatt ultraviolet (UV) laser 7 source in an operational high power proton accelerator facility. This paper reports in 8 detail the design, installation, and commissioning result of a macro-pulsed multi9 megawatt UV laser system and laser beam transport line for the laser stripping 10 experiment.

13 Keywords: Laser; Hydrogen ion; Laser stripping; Charge exchange injection; Spallation 14 Neutron Source

15

16 


\section{$1 \quad$ I. Introduction}

2 Advancements in laser technology have dramatically expanded the applications of lasers

3 to high energy physics [1]. Today, lasers have been used in a broad range of particle

4 accelerators from test stands to operational facilities. A number of important applications

5 with different levels of technical readiness have been demonstrated including beam

6 diagnostics [2], photo-injectors [3], Compton scattering-based light sources [4, 5], and

7 laser driven electron acceleration [6, 7]. Recently, a laser assisted hydrogen beam

8 stripping method was developed at the Spallation Neutron Source (SNS) [8]. The laser

9 stripping technique has the potential to make it possible to stack high-intensity proton

10 beam with no restrictions of beam loss and radiation activation at high beam intensities.

11 Following a successful proof-of-principle demonstration [9] using a Q-switched laser, a

12 more advanced experiment, or a proof-of-practicality experiment was recently proposed

13 by SNS [10] and funded by U.S. Department of Energy (DOE) High Energy Physics

14 Program. The goal of the project is to demonstrate high-efficiency $\mathrm{H}^{-}$beam stripping at

15 an operational accelerator setting. A typical neutron-production $\mathrm{H}^{-}$beam at SNS is

16 bunched in a macropulse (burst-mode) structure where each macropulse consists of 30-ps

17 micro-pulses repeating at $402.5 \mathrm{MHz}$.

18 Laser stripping imposes a number of challenges on both laser technology and laser

19 operation. First, high efficiency stripping requires lasers with high peak and high average

20 powers in the ultraviolet wavelength regime as well as a complicated macropulse

21 structure, which cannot be obtained using conventional chirped pulse amplification

22 (CPA) technology [11]. Such lasers are not available in the current laser market and

23 demand the development in macropulse amplification technology. Second, high radiation

24 in an operational high power proton accelerator puts significant restrictions on the

25 control/operation of the laser system. Besides, high intensity UV laser beam also

26 introduces risks to accelerator operation, such as the laser induced breakdown on the

27 vacuum windows. In most the above-mentioned applications [2-7], high power lasers

28 have been installed near electron accelerator beamlines where radiation concerns were

29 much insignificant compared to proton accelerators. 
1 In this paper, we describe the design and performance of a macropulse laser system that

2 provides picosecond pulses with multi-MW peak power at $355 \mathrm{~nm}$. The pulses are

3 repeating at $402.5 \mathrm{MHz}$ and are bunched into a burst mode structure with variable

4 macropulse durations and frequencies. The general design concept can be adapted to any

5 temporal beam structures in most accelerators. We also describe the implementation of

6 laser transport line (LTL) that delivers UV beam over a 60-meter long complicated beam

7 path with remote control/monitor of laser parameters. The LTL delivered UV beam to the

8 laser stripping location at a 70\% efficiency and survived high radiation doses induced by

9 megawatt proton beam accelerator. A laser parameter scalability to the full-cycle laser

10 stripping, i.e., $1 \mathrm{~ms}$ macropulse at $60 \mathrm{~Hz}$, is also discussed.

\section{2. Macropulse UV Laser}

\subsection{Laser parameter requirement}

14 The SNS laser stripping scheme consists of three steps: First, $\mathrm{H}^{-}$ions are converted to $\mathrm{H}^{0}$ 15 by stripping off the first electron in a magnetic field; then $\mathrm{H}^{0}$ atoms are excited from the 16 ground state $(n=1)$ to an upper level, i.e. $n=3$, by a laser; finally the excited states $\mathrm{H}^{0^{*}}$ 17 are converted to $\mathrm{H}^{+}$by stripping the second electron in a second magnetic field [8]. The 18 bandgap energy between the ground state and the $n=3$ state is $12.1 \mathrm{eV}$ which 19 corresponds to a laser wavelength of $102.6 \mathrm{~nm}$. Although some lasers, e.g. excimer lasers, 20 are emitting at the close wavelengths, those lasers are usually operating at continuous21 wave (CW) mode with relatively low power. Meanwhile, by taking advantage of the fact 22 that the hydrogen atoms are moving at a relativistic speed, it was shown in [12] that the 23 necessary photon energy to excite $\mathrm{H}^{0}$ atoms can be greatly reduced. In the case of SNS 24 linear accelerator, the $\mathrm{H}^{-}$beam reaches a kinetic energy close to $1 \mathrm{GeV}$ (the 25 corresponding $\mathrm{H}^{-}$ions and $\mathrm{H}^{0}$ atoms speed are near $87 \%$ of the light speed), the 26 wavelength of the laser light in the $\mathrm{H}^{0}$ atom rest frame can be lowered to around $355 \mathrm{~nm}$

27 if the laser and $\mathrm{H}^{0}$ beams interact with each other at a certain angle. Such a wavelength 28 can be readily achieved by tripling the frequency of an Nd:YAG laser that is one the most 29 popular high power pulsed light sources. 
1 While the three-step laser stripping concept [12] was proposed several decades ago, a

2 fundamental technical challenge was to deal with the Doppler broadening of the 3 hydrogen absorption linewidth due to the finite momentum spread of the $\mathrm{H}^{-} / \mathrm{H}^{0}$ beam.

4 Danilov [8] proposed an efficient method to excite all the hydrogen atoms nearly 5 simultaneously by using the Doppler dependence of the hydrogen rest-frame laser 6 frequency. By preparing a diverging laser beam, the interaction angle between the laser 7 and particle beams continuously changes across the hydrogen beam path and accordingly

8 the frequency of the light in the $\mathrm{H}^{0}$ atom rest frame decreases as the angle increases. The 9 diverging laser beam introduces an effective frequency "sweep" as the hydrogen beam

10 traverses the laser interaction region, which assures that all atoms with different energies 11 will be excited.

12 Figure 1(a) shows the calculated stripping efficiency as a function of the laser peak power.

13 Obviously, the stripping efficiency rapidly grows in proportional to the laser power at 14 low power levels while saturates quickly when the laser peak power exceeds $1 \mathrm{MW}$. The 15 stripping efficiency also depends on the laser beam divergence angle in the interaction 16 plane (assumed in the horizontal plane) and the laser beam size in the perpendicular 17 (vertical) plane, as shown in Fig. 1(b). To obtain stripping efficiency of 90\% and above, 18 we need to prepare a UV laser beam with a peak power of $1 \mathrm{MW}$, typical (horizontal) 19 divergence angle (RMS) around $0.5 \mathrm{mrad}$ and (vertical) beam size around $0.2 \mathrm{~mm}$ (RMS).

20 Temporal structure of the laser beam is another important factor. To minimize the 21 (average) laser power requirement, the laser beam needs to have the same temporal 22 structure as that of the ion beam. The baseline $\mathrm{H}^{-}$beam of the SNS accelerator has a $1 \mathrm{~ms}$ 23 pulse length and a repetition rate of $60 \mathrm{~Hz}$. Each macropulse contains micro-pulses 24 bunched by the SNS RFQ at a frequency of $402.5 \mathrm{MHz}$. For the laser stripping 25 experiment, the pulse width of the micro-pulses is compressed to $\sim 27 \mathrm{ps}$ (FWHM).

26 The goal of the present experiment is to verify the practicality of laser stripping by 27 demonstrating that all individual micro-pulses within a macropulse can be simultaneously 28 stripped at $~ 90 \%$ efficiency using a properly designed macropulsed UV laser. The 29 macropulse duration is set at $10 \mu$ s in the current experiment. The macropulse duration 30 can be expanded to $1 \mathrm{~ms}$ by changing the pump scheme in the proposed amplifier system. 
1 Maintaining the MW peak power over $1 \mathrm{~ms}$ is beyond the capability of the present laser

2 technology. On the other hand, by using the power recycling optical cavity, the proposed

3 laser stripping technology is scalable to full-cycle $\mathrm{H}$ - beam. A discussion on the

4 scalability will be given in the later part of this paper.

$5 \quad 2.2$ Macropulse laser system

6 Figure 2 shows a schematic of the stripping laser system. The laser adopts a master 7 oscillator power amplifier (MOPA) configuration. Primary segments include a fiber seed

8 laser, a macro-pulse generator, multiple-stage Nd:YAG amplifiers, and two harmonic

9 conversion crystals. The seed laser, i.e., the master oscillator, is an actively mode-locked

10 fiber laser pumped by $1480 \mathrm{~nm}$ diode lasers. The gain medium is a Ytterbium-doped fiber

11 which supports generation of laser light around $1064 \mathrm{~nm}$. The laser wavelength is

12 controlled via a temperature tuned fiber Bragg grating (FBG) with a tuning factor of 13.3

$13 \mathrm{pm} /{ }^{\circ} \mathrm{C}$. The temperature of $\mathrm{FBG}$ is stabilized at $36.0 \pm 0.05^{\circ} \mathrm{C}$ so that the corresponding 14 wavelength $1064.45 \mathrm{~nm}$ maximizes the Nd:YAG amplifier gain. The laser cavity (fiber 15 loop) is also temperature stabilized and the default cavity length corresponds to a round16 trip frequency of $\sim 4.43 \mathrm{MHz}$. An electro-optic modulator (EOM) modulates the loss in 17 the laser cavity for the active mode-locking. The phase lock loop circuit in the laser 18 provides a control signal to adjust the cavity length through a piezo-transducer (PZT) so 19 that its round-trip frequency matches a sub-harmonic of the pulse repetition frequency.

20 Fig. 3 shows the measured pulse width as a function of (a) the bias voltage $\left(V_{b}\right)$ of the 21 EOM and (b) phase loop control voltage $\left(V_{p h}\right)$ of the seed laser. Stable mode locking is 22 obtained over a quite large range of both parameters. The pulse width shows a sensitive 23 dependence on $V_{b}$, which provides an efficient way of pulse width tuning within $55-85$ 24 ps. The radio-frequency (RF) modulation signal on the EOM is synchronized to an 25 external timing signal at $402.5 \mathrm{MHz}$ with a synchronization accuracy of about $1 \mathrm{ps}$. The 26 seed laser also has a built-in fiber amplifier which brings the seed laser output power to $27200 \mathrm{~mW}$.

28 The infrared (IR) light pulses from the seed laser have a very low peak power $(\sim 10 \mathrm{~W})$.

29 To generate MW peak power at $355 \mathrm{~nm}$ required by the laser stripping, an amplification 30 factor of $\sim 10^{6}$ on the IR pulses from the seed laser is needed. In this work, the 
1 amplification is performed in a burst mode by using solid-state amplifiers. Both the 2 macropulse generation and amplification are conducted in free space. An acousto-optic 3 modulator $(\mathrm{AOM})$ is employed to generate macropulses with adjustable pulse durations,

4 repetition rates, and most importantly arbitrary pulse shapes. The macropulse shaping 5 capability is crucial to combat the effects of gain saturation in the amplifier chain. Since 6 the pulse being amplified is short compared to the lamp pulse, the first part of the pulse 7 envelope sees the highest gain and the gain is depleted as the pulse envelope passes 8 through the amplification rods. To achieve a flat macropulse of the UV light, the 9 macropulse of the seed light is controlled so that the front of the pulse envelope has less 10 energy than the end of the pulse envelope, thus compensating for this gain depletion. The 11 AOM is driven by a voltage controlled RF amplifier at a fixed frequency close to the 12 resonance frequency ( $41 \mathrm{MHz}$ ) of the crystal while the amplitude of the RF signal is 13 controlled by an arbitrary waveform generated on a computer. For the present stripping

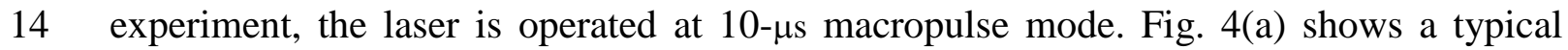
15 macropulse waveform of the UV beam with the peak power of $\sim 2 \mathrm{MW}$. The 16 corresponding control voltage waveform on AOM is shown in Fig. 4(b).

17 Our custom-built macropulse amplifier has three stages. Each stage consists of a pair of 18 identical Nd:YAG rods with the diameters of 5-, 6-, and 9-mm for amplifier stages 1, 2, 19 and 3, respectively. Both the 5-mm and 6-mm amplifiers are single flash lamp pumped 20 with the first rod pumped from the top and the second rod pumped from the bottom. The 21 9-mm amplifiers are pumped by two flash lamps from sides. In each amplifier stage, 22 there are two compensation optics between the two rods: a negative lens for thermal 23 lensing compensation and a quartz rotator for thermal birefringence compensation. The 24 amplified IR macropulses are converted to the UV light using a $25 \mathrm{~mm}$ long LBO 25 doubler and a $30 \mathrm{~mm}$ long LBO tripler. More details of the amplifier can be found in the 26 previous paper [13]. The amplifier was designed to generate $1 \mathrm{MW}$ (peak power) UV 27 pulses bunched in 10-us@10-Hz macropulses. We have implemented a number of 28 improvements in recent years. Maximum gains of the first two amplifier stages have been 29 achieved after proper optics realignment and insertion of an additional isolator between 30 5- and 6-mm rods. The image relay optics and spatial optical filters have been redesigned 31 to maximize the amplifier output power while maintaining high beam quality. The 
1 improvements boosted the maximum peak power of the UV pulses to $\sim 3.5 \mathrm{MW}$, more

2 than three times the design specification.

3 Figure 5 shows the IR micro-pulses measured using a high bandwidth photo-detector

4 (New Focus 1444). A typical micro-pulse waveform is shown in the inset box. The pulse

5 width varies from 55 to $85 \mathrm{ps}$ and the temporal jitter is about $1 \mathrm{ps}$. Accurate information

6 of the UV pulse width is important in the optimization of pulse energy in the

7 amplification. We have developed a multifunctional optical correlator to measure the

8 pulse width of the UV beam without using any external reference pulse [14]. The

9 correlator measures both auto-correlation between IR pulses and cross-correlation

10 between IR and UV pulses using the same nonlinear optical crystal. We were able to

11 perform a detailed characterization of the UV beam at a variety of amplifier parameters.

12 Fig. 6 shows the measured UV pulse width as a function of the IR pulse width. We also

13 calculated the UV pulse peak power using the measured UV pulse width and UV

14 (average) power. While the UV pulse width is nearly proportional to the IR pulse width,

15 the peak power shows a nonlinear inverse dependence on the pulse width. This is because

16 the harmonic generation efficiency depends on the intensity of the incoming light. As a

17 result, when the seeder pulse width is narrowed from 84 to $54 \mathrm{ps}$, the UV pulse width

18 changes from $58 \mathrm{ps}$ to $34 \mathrm{ps}$ and the peak power of the UV light increases from 1.2 MW

19 to $3.1 \mathrm{MW}$.

20 The UV beam quality is mainly determined by the incoming beam size to the $9-\mathrm{mm}$

21 amplifier and the entire amplification factor (particularly the setting of the last amplifier).

22 To optimize the beam quality, we set up the 5- and 6-mm amplifiers at their maximum

23 voltages $(\sim 1.4 \mathrm{kV})$ while limiting the 9-mm amplifier to relatively low voltages (1.15-

$241.25 \mathrm{kV}$ ). Fig. 7(a) shows a near-field profile of the UV beam at $\sim 2 \mathrm{MW}$ and Fig. 7(b)

25 shows the far-field profile. The laser beam shows a TEM ${ }_{00}$ Gaussian mode in the far-field

26 and the $\mathrm{M}^{2}$ factor is measured to be $\sim 1.3$ in both horizontal and vertical directions. The

$27 \mathrm{M}^{2}$ factor increases when the peak power exceeds $3 \mathrm{MW}$.

29 3. Optical Setup for Stripping Experiment

$30 \quad 3.1$ Laser transport line 
1 Operating lasers in a proton accelerator facility always faces concerns of radiation

2 induced damage. Although in the previous laser stripping experiment [9] the laser was

3 located in the linac beam dump area, the accelerator was operating with beam powers at

4 the kilowatt level at the time. As the SNS accelerator was ramping up the proton beam

5 power, the residual radiation levels grew rapidly. It was experimentally verified that the

6 laser system (mainly the electronics parts) did not survive more than a few hours even in

7 a relatively lower radiation area. Currently, SNS is routinely operating at the $1 \mathrm{MW}$

8 proton beam power level. At this power level, the radiation doses are several orders of

9 magnitude higher than before. In particular, radiation in the Ring injection area reaches

10 tens of kilo-Rads.

11 To protect the laser from radiation induced damage, we located the macropulse laser

12 system in the Ring Service Building (RSB) and built an LTL to deliver the laser beam to

13 the stripping chamber. The RSB is about 10 meters above the beam line and shielded

14 from the accelerator tunnel through a 20-meter thick concrete wall. The only available

15 penetrations between the RSB and tunnel are 6-inch penetration chases which were

16 originally designed as cable chases. A schematic of the LTL is shown in Fig. 8. It consists

17 of three parts: the first part transports the laser beam from the laser table to the entrance

18 of the penetration hole in the RSB, the second part is a 70-ft long cable chase, and the last

19 part relays the laser beam from the exit of the penetration chase to the stripping chamber

20 location in the Ring/HEBT tunnel areas. The entire LTL is enclosed in aluminum tubes

21 and the entrance/exit of the LTL are sealed with 4-inch vacuum viewport windows that

22 are AR-coated at $355 \mathrm{~nm}$. The enclosure is important to eliminate air flow caused by

23 pressure/temperature difference between the RSB and accelerator tunnel.

24 Although the total length of the LTL is only about 60 meters, due to the existing 25 accelerator beam line, electronic and mechanical equipment, the available space for the

26 LTL is extremely limited and the resulting LTL has a very complicated path configuration

27 as shown in Fig. 8. A total of 8 mirrors have to be used to relay the beam in 8 different

28 planes. Table I lists the parameters of each relay mirror. Note that mirrors reflect light at

29 different angles varying from $\sim 6^{\circ}$ to $\sim 50^{\circ}$ are used due to the space limitation. All

30 mirrors are 3-inch dielectric mirrors with high-reflection (HR) coating at $355 \mathrm{~nm}$ over a 
1 wide range of reflection angles $\left(5^{\circ}-53^{\circ}\right)$. In addition, most of mirrors are very difficult

2 to access due to radiation/electrical concerns. Therefore, all relay mirror mounts except

3 the first two are equipped with a pair of pico-motor driven actuators for remote beam

4 steering and a compact analog camera for beam position monitor. Two examples of the

5 mirror box implementation are shown Fig. 9.

6 Many conventional laser transport lines employ the image relay approach where beam is

7 focused and collimated between two relay mirrors. This approach turns out to be

8 impractical in the present environment due to the limitation of space for lens mount, lack

9 of control and accessibility. Instead, we chose an approach of propagating a collimated

10 laser beam through the entire LTL without image relay. A series of LTL simulations were

11 carried out on the optical table in the laser lab by using multiple reflections on 8 mirrors

12 to make up a path length close to 60 meters. Using LTL simulations, we compared

13 different telescope settings for beam collimation and studied the transmission efficiency

14 and final beam quality for each case. The optimal transmission performance is obtained

15 when the laser beam is focused close to the final destination. In this case, the laser beam

16 is collimated to have beam diameters of about $10-12 \mathrm{~mm}\left(1 / \mathrm{e}^{2}\right)$ along the LTL. The

17 transmission efficiency was estimated to be around 75\%. Major beam losses come from

18 the absorption/scattering on the mirror surfaces and loss of high-order modes of the laser

19 beam during the propagation through the LTL.

\subsection{Stripping experiment optics}

21 The laser stripping experiment is carried out at $\sim 20$ meters upstream of the SNS ring

22 injection area. As described in the previous section, successful stripping requires a

23 narrow parameter range in laser beam size, divergence angle, and interaction angle

24 between the laser and ion beams. In addition, to avoid possible optical breakdown on the

25 vacuum windows, the minimum beam sizes on the entrance and exit vacuum windows

26 have to be limited. Fig. 10 shows a schematic of the optical setup around the stripping

27 chamber. The telescope consists of a lens pair $\left(f_{1}=-100 \mathrm{~mm}\right.$ and $\left.f_{2}=200 \mathrm{~mm}\right)$ and the

28 spacing between the two lenses is controlled by a stepper motor. The location and

29 spacing of the telescope controls the beam size and divergence angle at the interaction

30 point. Limit switches are implemented at each end of the motor to restrict the beam sizes 
1 on the vacuum windows. The interaction angle is controlled by a steering mirror and a

2 second stepper motor. The entrance and exit windows are located at $\sim 40$ inches from the

3 focused point of the laser beam by using extension tubes. Laser beam positions are

4 monitored before and after the stripping chamber using two Gigabit Ethernet cameras

5 (Cam1 and Cam2 in Fig. 10). The laser power is monitored also by an Ethernet accessed

6 power meter.

7 Table II summarizes the major laser parameters measured before the stripping experiment.

8 The laser beam is interacting with a $1 \mathrm{GeV} \mathrm{H}$ - beam at $37.5^{\circ}$. At the interaction point, the

9 designed laser beam has a full-width half-maximum (FWHM) beam divergence angle of

$101.2 \mathrm{mrad}$ and a FWHM beam size of $0.5 \mathrm{~mm}$. The transmission efficiency was measured

11 to be near $70 \%$ which is close to our lab simulations.

\subsection{Laser operation and control}

13 Like many other experiments in the accelerator facilities, laser stripping experiments are

14 conducted in the central control room, which requires high level of automation and

15 remote access/manipulation of laser and optical parameters. Table III lists major

16 electronic chassis and computers used in the operation. Most controls are realized in the

17 extensible display manager (EDM) screen during the lase stripping experiment.

18 The customized macropulse laser amplifier has a sophisticated graphic user interface

19 (GUI) software that allows the users to operate the laser through a computer. Using the

20 accelerator network, one can remotely turn on/off the laser operation, change amplifier

21 settings, switch between different macropulse modes, and monitor the status of amplifiers

22 and optical crystals. The laser power is also remotely controlled using a motor driven

23 rotational stage to control an optical wave plate. There are two levels of temporal

24 synchronization. The micro pulses generated in the seed laser are synchronized to the

$25402.5 \mathrm{MHz}$ RF timing of the SNS accelerator. The phase difference can be remotely

26 tuned using a computer controlled digital phase shifter with a precision of 0.1 degree

27 (corresponding to $\sim 0.7 \mathrm{ps}$ ). The laser macropulse is timed to the accelerator according to

28 a beam position monitor near the stripping chamber and its phase is also computer

29 controlled to nanosecond accuracy. 
1 Beam steering in the LTL is implemented using 7 pairs of pico-motor actuators. The

2 Thorlabs open-loop actuators have a very high radiation tolerance and are controlled by a

3 single computer. The same computer also controls two stepper motors in front of the

4 stripping chamber (Fig. 10) to change the laser beam size/divergence angle at the

5 interaction point.

6 Laser beam positions along the LTL are monitored using Watec analog cameras toward

7 the surface of the mirror. The tiny cameras were chosen since they have reasonably high

8 radiation tolerance, and are easy to install in a limited space. All images are displayed on

9 a monitor next to the laser table to facilitate the alignment. The laser beam positions right

10 before and after the stripping chamber are monitored by two CMOS cameras (Allied

11 Vision GC 750). Both cameras are synchronized to the $10 \mathrm{~Hz}$ macropulse timing and the

12 captured images are sent back to the computer through Ethernet network. The images are

13 then analyzed to extract the centroid position which is used to feedback control the piezo-

14 transducer (PZT) mirror mounted right before the LTL on the laser table. The feedback

15 control is very effective in the reduction of slow $(<1 \mathrm{~Hz})$ drifts of the laser beam through

16 the LTL [15].

17 Finally, the laser power after the stripping chamber is measured by a power meter. The

18 measured power determines the actual laser pulse energy in the stripping chamber by

19 taking into account the losses in vacuum windows.

\section{Discussions}

22 The present laser stripping experiment has been successfully conducted using a 10-us

23 laser macropulse. A stripping over 10 mini-pulses of the $\mathrm{H}$ - beam has been observed with

24 the maximum stripping efficiency close to 99\%. Details of experimental results are 25 reported in [16]. Here we briefly discuss the scalability of the present laser stripping 26 scheme to a full-cycle $\mathrm{H}^{-}$beam, e.g. 1-ms@60-Hz in the case of SNS neutron production 27 operation.

28 It is clear that the flash lamp pumping scheme in the present laser amplifier is the major 29 limiting factor of the macropulse structure. Typical flash lamps limit the repetition rate to $3030 \mathrm{~Hz}$ and the pulse duration to $\sim 200 \mu$ s. The flash lamp limitation can be resolved by 
1 using a diode pump scheme. A commercially available 3-stage diode-pumped solid-state

2 laser amplifier can generate multi-millisecond macro pulses [17]. A less obvious limiting

3 factor, however, is the strong dependence of the achievable UV light power on the

4 macropulse duration. In our experiment, we observed that the achievable peak power of

5 the IR pulses rapidly dropped below $1 \mathrm{MW}$ when the macropulse duration was increased

6 beyond a few tens of microseconds. In this case, the peak power of the UV pulses after

7 harmonic conversion is at the $10 \mathrm{~kW}$ level, which is two orders of magnitude lower than

8 the required power for laser stripping.

9 On the other hand, since the cross-section number in the photon-particle interaction in the

10 laser stripping process is extremely small, the power loss of the laser light is negligible.

11 The laser power can be recycled if the laser stripping is located in an optical cavity.

12 External optical cavities have been routinely applied to recycle the power from single-

13 frequency lasers or mode-locked lasers which have pico-/femto-second pulses repeating

14 at tens of $\mathrm{MHz}$ to $\mathrm{GHz}$. However, for a burst-mode laser, due to a very small duty factor

15 and low repetition rate of the burst, it is impossible to generate an effective error signal

16 within the short duration of the burst. In such a case, the conventional cavity locking

17 technique will fail and a different cavity locking method is demanded. At SNS, we

18 proposed a double-resonance optical cavity (DROC) scheme and developed a robust

19 locking scheme to realize cavity enhancement of burst mode laser pulses. In the prototype

20 experiment, we have shown how a Fabry-Perot based DROC can be simultaneously

21 locked to an infrared (IR) and its third-harmonic ultraviolet picosecond pulses using a

22 frequency shift technique. We have experimentally demonstrated that such a cavity can

23 be applied to enhance burst-mode UV laser pulses with arbitrary burst lengths and

24 repetition rates [18]. The cavity enhancement of UV macropulses was achieved with an

25 enhancement factor of 50. By using diode-pumped solid state amplifier and optical cavity

26 enhancement technology, it is promising to scale the present laser parameters to fulfill the

27 requirement of the full-cycle stripping experiment.

\section{Conclusion}


1 We have described the design and commissioning of a macropulse laser system and its

2 transport line for the laser assisted $\mathrm{H}^{-}$beam stripping experiment conducted at the proton

3 accelerator of the Spallation Neutron Source. The macropulse laser has a MOPA

4 configuration and consists of a mode-locked picosecond pulsed seed laser and a burst-

5 mode Nd:YAG laser amplifier. We have achieved UV pulses with the pulse widths

6 varying between 34 to $54 \mathrm{ps}$ and a maximum peak power over $3.5 \mathrm{MW}$. A laser transport

7 line is installed to deliver the UV beam to the laser stripping chamber. The LTL has

8 capabilities such as a remote control and monitor of laser parameters including phase

9 delay, beam power, beam size, beam divergence, and interactions angle. A transmission

10 efficiency of $70 \%$ has been achieved. A successful stripping with $>90 \%$ efficiency has

11 been demonstrated over a $10 \mu$ s macropulse by using the developed laser and optical

12 system [16]. We have also discussed the scalability of the present experiment to the

13 parameters required for the full-cycle laser stripping.

\section{Acknowledgements}

16 We acknowledge C. Huang and Y. Takeda for their contributions to the laser and optical

17 system design and diagnostics, J. Diamond and S. Murray III for their technical helps

18 during installation. ORNL is managed by UT-Battelle, LLC, under contract DE-AC05-

19 00OR22725 for the U.S. Department of Energy. This research was supported by the

20 DOE Office of Science, Basic Energy Science, Scientific User Facilities. The work has

21 also been supported by U.S. DOE grant DE-FG02-13ER41967. 


\section{References}

3 [1] G.A. Mourou, T. Tajima, and S.V. Bulanov, "Optics in the relativistic regime," Rev. 4 Mod. Phys. 78 (2006) 309.

5 [2] Y. Honda, N. Sasao, S. Araki, H. Hayano, Y. Higashi, K. Kubo, T. Okugi, T. 6 Taniguchi, N. Terunuma, J. Urakawa, Y. Yamazaki, K. Hirano, M. Nomura, M. Takano, 7 and H. Sakai,, "Measurements of electron beam emittance in the Accelerator Test Facility 8 damping ring operated in multibunch modes," Phys. Rev. ST Accel. Beams 6, (2003) 9092802.

10 [3] I. Will, G. Koss, I. Templin, “The upgraded photocathode laser of the TESLA Test 11 Facility," Nucl. Instr. Meth. A, 541 (2005) 467.

12 [4] F. Ebina et al, "Laser pulse circulation system for a compact monochromatic hard X13 ray source," Nucl. Instr. and Meth. B 241 (2005) 905.

14 [5] K. Sakaue, M. Washio, S. Araki, M. Fukuda, Y. Higashi, Y. Honda, T. Omori, T. 15 Taniguchi, N. Terunuma, J. Urakawa, and N. Sasao, "Observation of pulsed x-ray trains 16 produced by laser-electron Compton scatterings,” Rev. Sci. Instrum. 80 (2009) 123304.

17 [6] W.P. Leemans, B. Nagler, A. J. Gonsalves, C. Toth, K. Nakamura, C. G. R. Geddes, E. 18 Esarey, C. B. Schroeder, and S. M. Hooker, "GeV electron beams from a centimetre-scale 19 accelerator,” Nature Phys. 2 (2006) 696.

20 [7] J. Faure, C. Rechatin, A. Norlin, A. Lifschitz, Y. Glinec, and V. Malka, "Controlled 21 injection and acceleration of electrons in plasma wakefildes by colliding laser pulses," $22 \quad$ Nature 444 (2006) 737.

23 [8] V. Danilov, A. Aleksandrov, S. Assadi, S. Henderson, N. Holtkamp, T. Shea, A. 24 Shishlo, Y. Braiman, Y. Liu, J. Barhen, and T. Zacahria, "Three-step H- charge exchange 25 injection with a narrow-band laser,” Phys. Rev. ST Accel. Beams 6 (2003) 053501.

26 [9] V. Danilov, A. Aleksandrov, S. Assadi, J. Barhen, W. Blokland, Y. Braiman, D. Brown, 27 C. Deibele, W. Grice, S. Henderson, J. Holmes, Y. Liu, A. Shishlo, A. Webster, and I. N. 28 Nesterenko, "Proof-of-principle demonstration of high efficiency laserassisted H- beam 29 conversion to protons," Phys. Rev. ST Accel. Beams 10 (2007) 053501. 
1 [10] S. Cousineau, A. Aleksandrov, V. V. Danilov, T. Gorlov, Y. Liu, A. Menshov, M.

2 Plum, A. Rakhman, A. Shishlo, N. Luttrell, F. Garcia, D. Johnson, Y. Wang, Y. Takeda,

3 "Status of preparations for a 10 microsecond laser-assisted $\mathrm{H}^{-}$beam stripping

4 experiment," Proc. IPAC 2014, Dresden, Germany, 1864 (2014).

5 [11] J. Limpert, F. Roser, D. N. Schimpf, E. Seise, T. Eidam, S. Hädrich, J. Rothhardt, C.

6 J. Misas, and A. Tünnermann, "High repetition rate gigawatt Peak power fiber laser

7 systems: challenges, design, and experiment”, IEEE JSTQE 15 (2009) 159.

8 [12] I. Yamane, in Summary Report of Session K on H- Stripping, Proceedings of the

9 20th ICFA Advanced Beam Dynamics Workshop on High Intensity and High Brightness

10 Hadron Beams, Batavia, IL, 2002, AIP Conf. Proc. No. 642 (AIP, New York, 2002).

11 [13] C. Huang, C. Deibele, and Y. Liu, "Narrow linewidth picosecond UV laser with 12 mega-watt peak power", Opt. Express 21 (2013) 9123.

13 [14] A. Rakhman, Y. Wang, F. Garcia, C. Long, C. Huang, Y. Takeda, Y. Liu, 14 "Multifunctional optical correlator for picosecond ultraviolet laser pulse measurement," 15 Appl. Opt. 53 (2014) 7603.

16 [15] R. Hardin, Y. Liu, C. Long, A. Aleksandrov, "Active beam position control of high 17 power pulsed laser for laser-based remote diagnostics," Opt. Express 19 (2011) 2874.

18 [16] S. Cousineau, M. Kay, A. Rakhman, M. Plum, T. Gorlov, Y. Liu, A. Aleksandrov, 19 and A. Shishlo, "First demonstration of laser-assisted H- charge exchange for 20 microseconds duration beams," submitted for publication.

21 [17] M. N. Slipchenko, J. D. Miller, S. Roy, T. R. Meyer, J. G. Mance, and J. R. Gord, 22 "100 kHz, $100 \mathrm{~ms}, 400 \mathrm{~J}$ burst-mode laser with dual-wavelength diode-pumped 23 amplifiers," Opt. Lett. 39 (2014) 4735.

24 [18] A. Rakhman, M. Notcutt, and Y. Liu, "Power enhancement of burst-mode ultraviolet 25 pulses using a doubly resonant optical cavity", Opt. Lett. 40 (2015) 5562. 


\section{$1 \quad$ Figure captions}

3 Figure 1 Stripping efficiency as a function of (a) laser peak power and (b) laser beam size

4 and divergence angle.

5 Figure 2 Schematic of macropulse laser system. EOM: electro-optic modulator, AOM:

6 acousto-optic modulator, PZT: piezo-electric transducer, $\lambda / 2$ : half-wave plate, SPF:

7 single-mode polarization-maintaining fiber, SF: spatial filter, SHG: second-harmonic

8 crystal, THG: third-harmonic crystal.

9 Figure 3 Seed laser parameter investigation results. (a) Pulse width vs. EOM bias voltage;

10 (b) Pulse width vs. phase control voltage of mode-lock loop. 'U' indicates parameter

11 regions where stable mode-lock cannot be obtained.

12 Figure 4 (a) 10- $\mu$ s macropulse waveform. (b) Control waveform on AOM.

13 Figure 5 A typical IR micro pulse waveform detected by a fast photodiode. The inset box

14 shows zoom-in pulse shape of a micro pulse.

15 Figure 6 UV pulse width and peak power vs. IR pulse width measured by the multi-

16 functional optical correlator.

17 Figure 7 Spatial profiles of UV beam. (a) Near-field and (b) far-field patterns.

18 Figure 8 Schematic of LTL. (a) LTL in the RSB is linked to (b) LTL in the Ring through a

19 20-meter long chase indicated by $\mathrm{A}-\mathrm{A}^{\prime}$ in the figure. M1 - M8 are mirrors for relay.

20 Figure 9 Examples of optics boxes in the laser transport line.

21 Figure 10 Optical setup around the laser stripping chamber in the accelerator tunnel. 
Table I LTL Components

\begin{tabular}{|l|l|c|l|}
\hline \multicolumn{1}{|c|}{ Mirror } & \multicolumn{1}{|c|}{ Description } & Reflection angle & \multicolumn{1}{c|}{ Propagation length (cm) } \\
\hline Mirror \#1 & $\begin{array}{l}\text { LTL Box \#1 (RSB) } \\
\text { No camera, No picomotor }\end{array}$ & $52^{\circ}$ & 110 \\
\hline Mirror \#2 & $\begin{array}{l}\text { LTL Box \#2 (RSB) } \\
\text { No camera, No picomotor }\end{array}$ & $45^{\circ}$ & 527 \\
\hline Mirror \#3 & $\begin{array}{l}\text { LTL Box \#3 (RSB) } \\
\text { Pico-motors (2), camera }\end{array}$ & $45^{\circ}$ & 122 \\
\hline Mirror \#4 & $\begin{array}{l}\text { LTL Box \#4 (RSB) } \\
\text { Pico-motors (2), camera }\end{array}$ & $35^{\circ}$ & 2339 \\
\hline Mirror \#5 & $\begin{array}{l}\text { LTL Box \#5 (Ring tunnel) } \\
\text { Pico-motors (2), camera }\end{array}$ & $5.6^{\circ}$ & 50 \\
\hline Mirror \#6 & $\begin{array}{l}\text { LTL Box \#5 (Ring tunnel) } \\
\text { Pico-motors (2), camera }\end{array}$ & $16.9^{\circ}$ & 311 \\
\hline Mirror \#7 & $\begin{array}{l}\text { LTL Box \#6 (Ring tunnel) } \\
\text { Pico-motors (2), camera }\end{array}$ & $50.3^{\circ}$ & 135 \\
\hline Mirror \#8 & $\begin{array}{l}\text { LTL Box \#7 (Ring tunnel) } \\
\text { Pico-motors (2), camera }\end{array}$ & $45^{\circ}$ & 2439 \\
\hline
\end{tabular}

4 


\begin{tabular}{|c|c|c|}
\hline & Required & Delivered \\
\hline Macro-pulse length & $10 \mu \mathrm{s}$ & $12 \mu \mathrm{s}$ \\
\hline Micro-pulse width & $>30 \mathrm{ps}$ & $30-50$ ps (adjustable) \\
\hline Peak power & $1.5 \mathrm{MW}$ & 4.0 MW (at pulse width $35 \mathrm{ps}$ ) \\
\hline LTL transmission efficiency & $>60 \%$ & $70 \%$ \\
\hline Maximum power at IP & $>1 \mathrm{MW}$ & 2 MW@ 33 ps \\
\hline Horizontal beam divergence $(4 \sigma)$ at IP & $1.6-2.6 \mathrm{mrad}$ & $2.3-3.0 \mathrm{mrad}$ \\
\hline Vertical beam size $(4 \sigma)$ at IP & $1.0-1.4 \mathrm{~mm}$ & $1.1 \mathrm{~mm}$ \\
\hline Pointing stability at the IP & & $\pm 0.10 \mathrm{~mm}(\mathrm{H}) \mathrm{x} \pm 0.11 \mathrm{~mm}(\mathrm{~V})$ \\
\hline $\begin{array}{l}\text { Maximum peak laser intensity on } \\
\text { entrance vacuum window }\end{array}$ & $\leq 100 \mathrm{MW} / \mathrm{cm}^{2}$ & $57.9 \mathrm{MW} / \mathrm{cm}^{2} *$ \\
\hline $\begin{array}{l}\text { Maximum peak laser intensity on exit } \\
\text { vacuum window }\end{array}$ & $\leq 100 \mathrm{MW} / \mathrm{cm}^{2}$ & $78.6 \mathrm{MW} / \mathrm{cm}^{2} *$ \\
\hline
\end{tabular}

4 *These values were measured on $1 \mathrm{MW}$ peak laser power. At the end of the experiment, 5 the laser power was raised to $2 \mathrm{MW}$. No laser induced breakdown was observed over 8 6 hours of influence. 


\section{Table III Control electronics}

2

\begin{tabular}{|l|l|}
\hline Control Unit & \multicolumn{1}{c|}{ Description } \\
\hline IOC 1 & Laser control (remote on/off, amplifier voltage setting) \\
\hline IOC 2 & $\begin{array}{l}\text { Laser power control } \\
\text { 6 steering mirrors } \\
\text { Interaction angle control } \\
\text { Laser beam divergence angle control } \\
\text { Laser beam size control } \\
\text { Horizontal position control }\end{array}$ \\
\hline IOC 3 & $\begin{array}{l}\text { Laser beam pointing stability feedback control using } \\
\text { PZT driven mirror }\end{array}$ \\
\hline IOC 4 & Laser beam position sensing using GigE cameras \\
\hline IOC 5 & Laser power monitor after the stripping chamber \\
\hline
\end{tabular}

3

4

5

6

7

8

9

10

11

12

13

14

15

16

17

18 
(a)

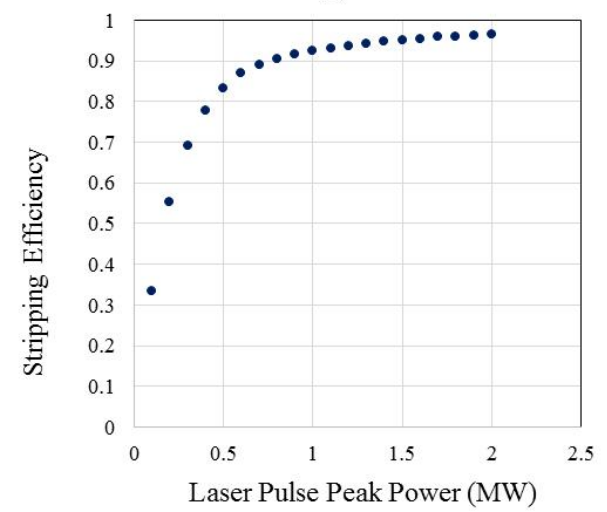

(b)

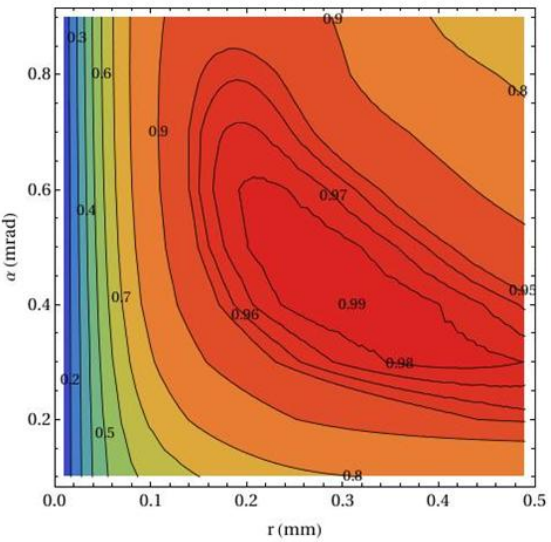

Figure 1

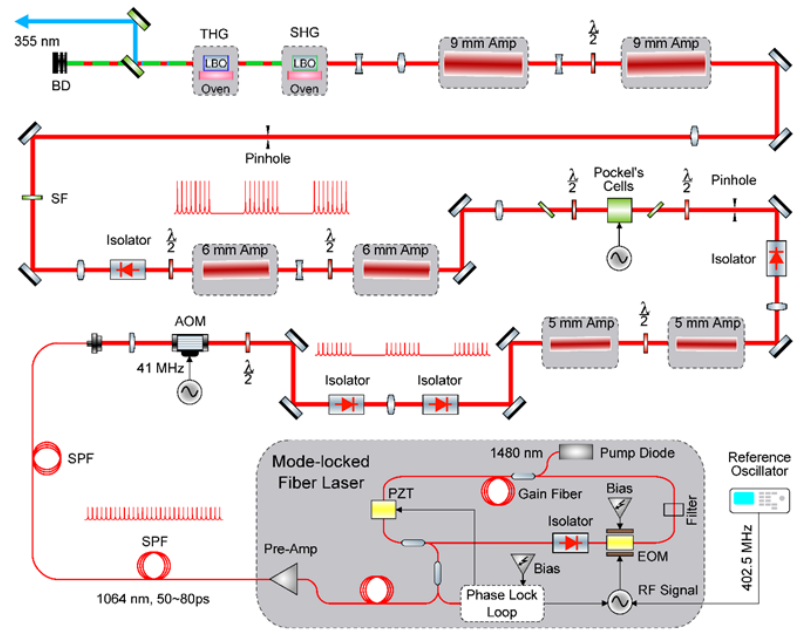


(a)
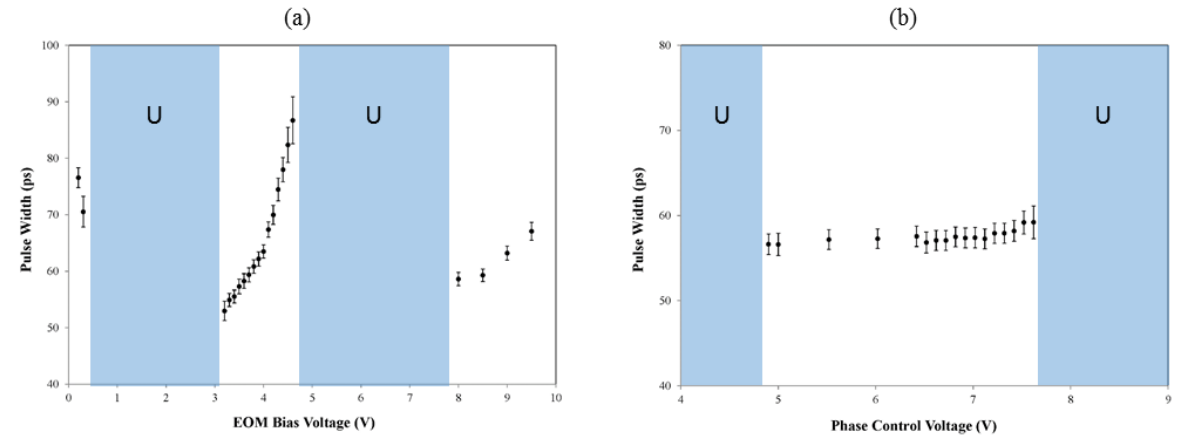

Figure 3

1

2
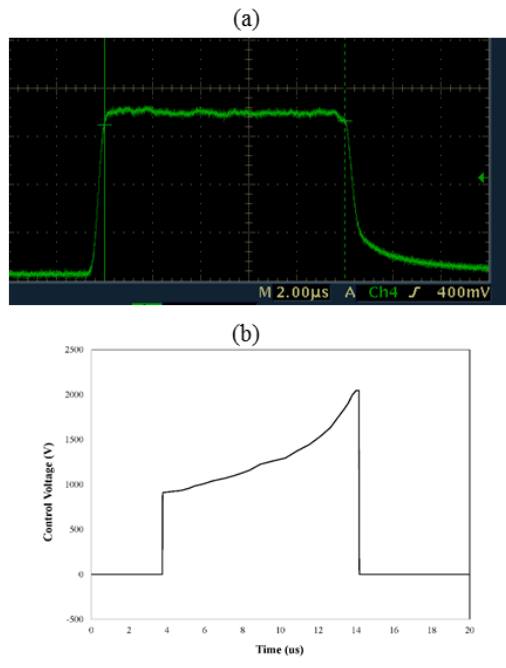

3

Figure 4

4 


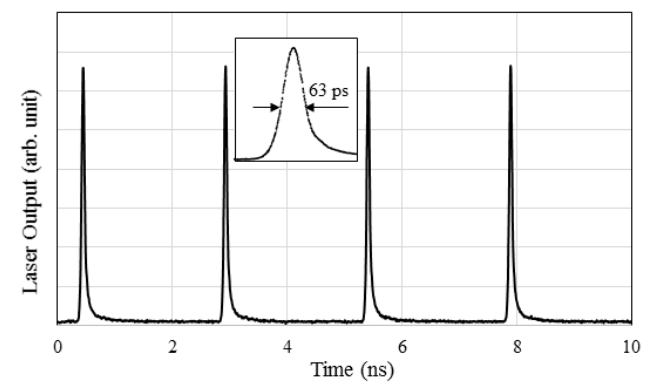

Figure 5

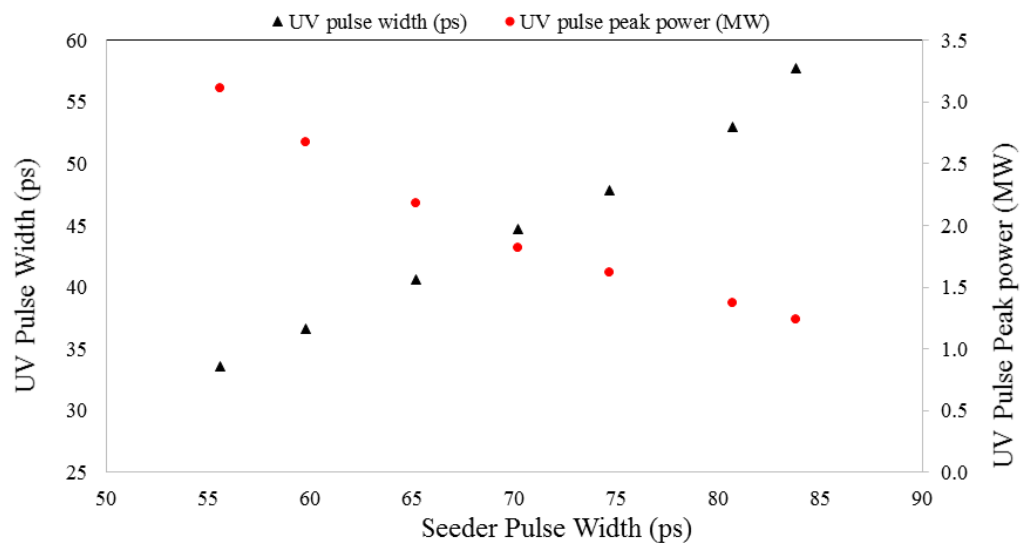

4

Figure 6 
(a)
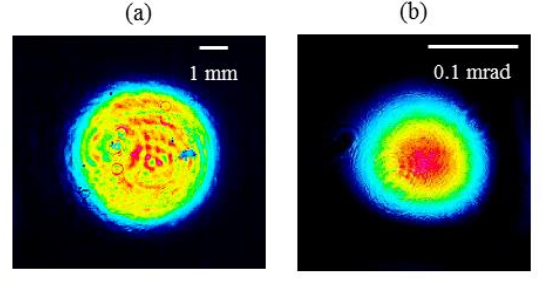

1

2
Figure 7

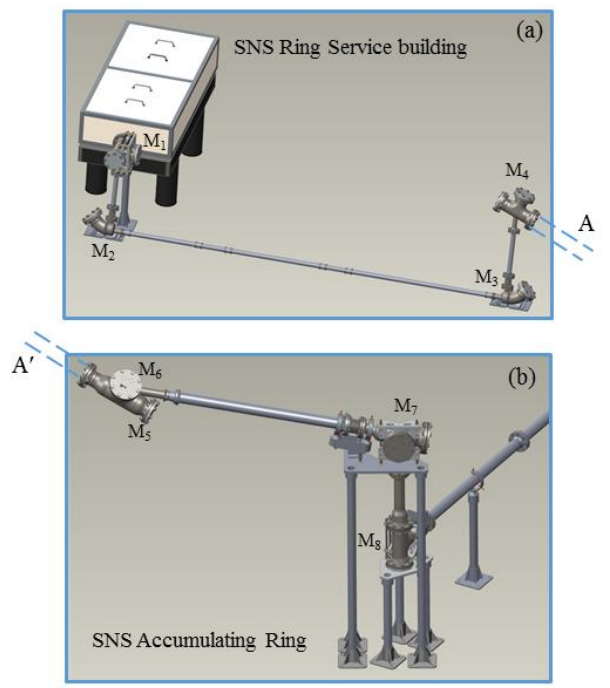

Figure 8 
(a)
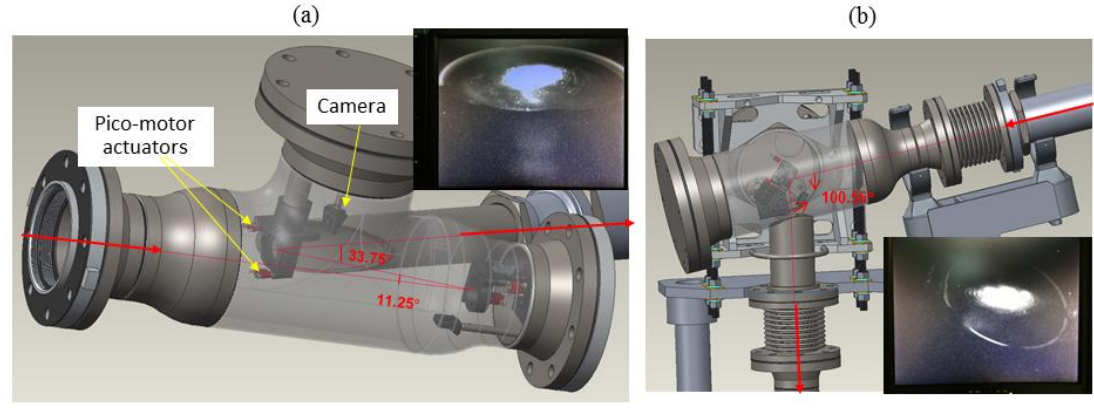

Figure 9

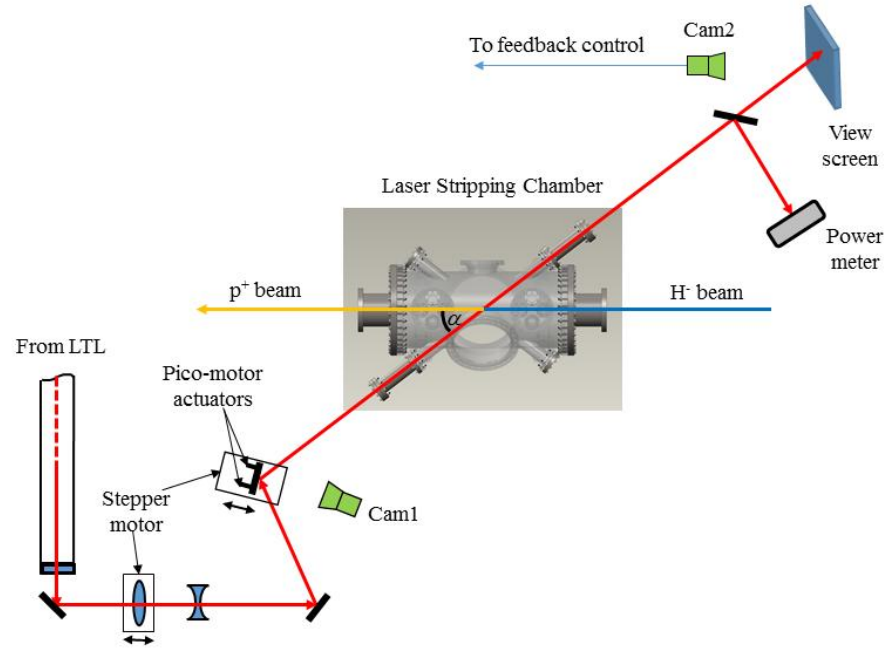


(a)

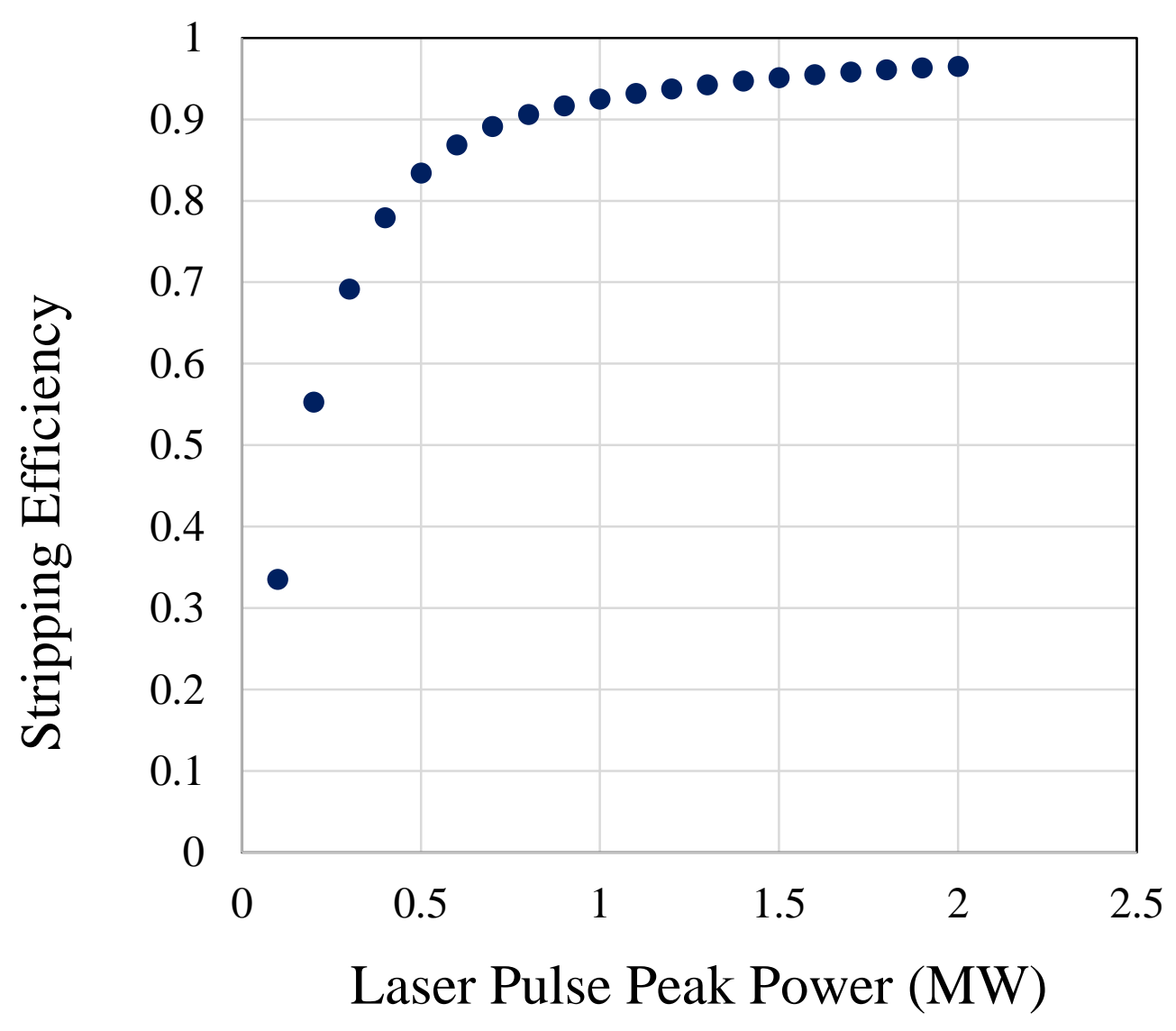

(b)

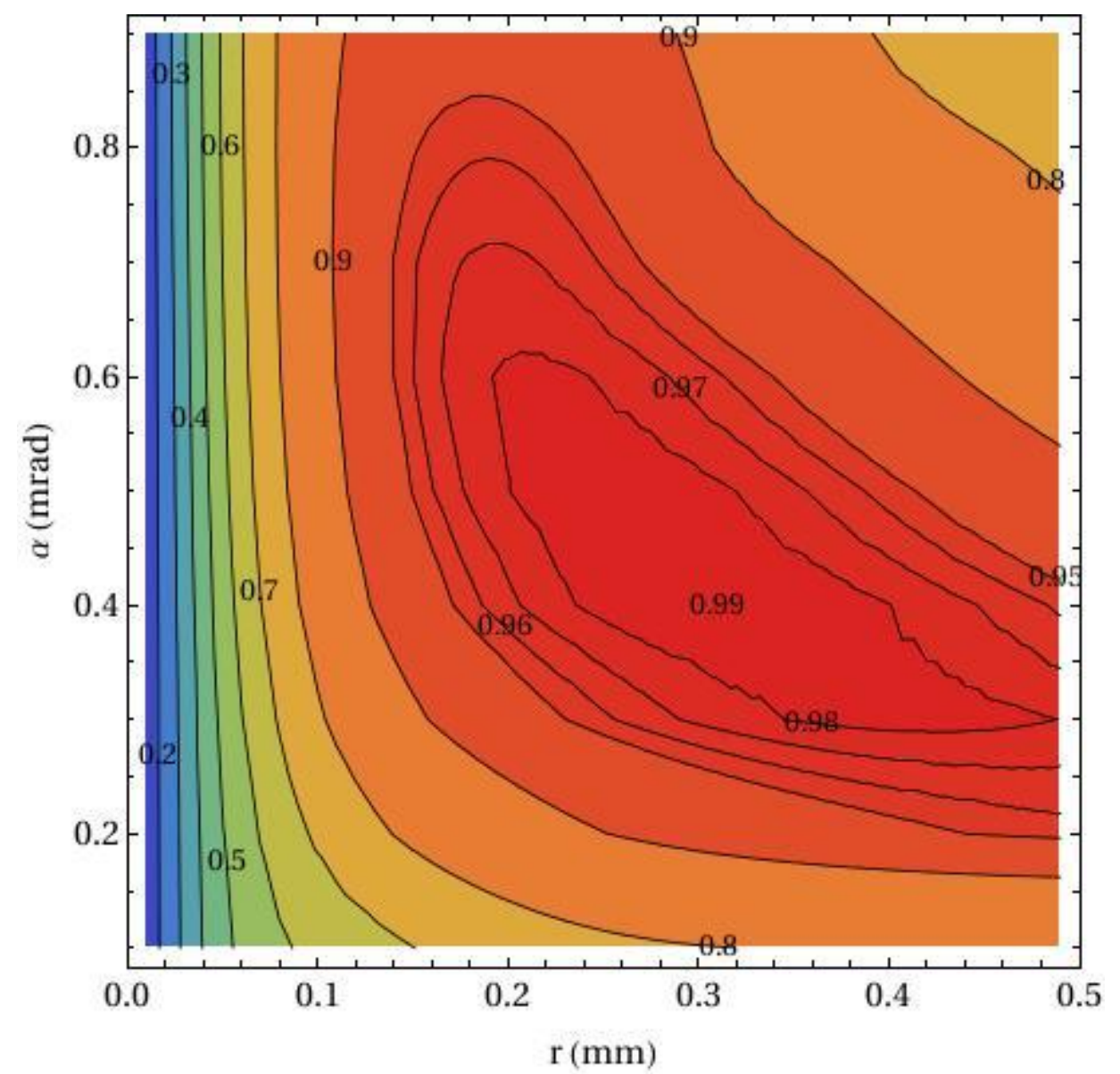

Figure 1 


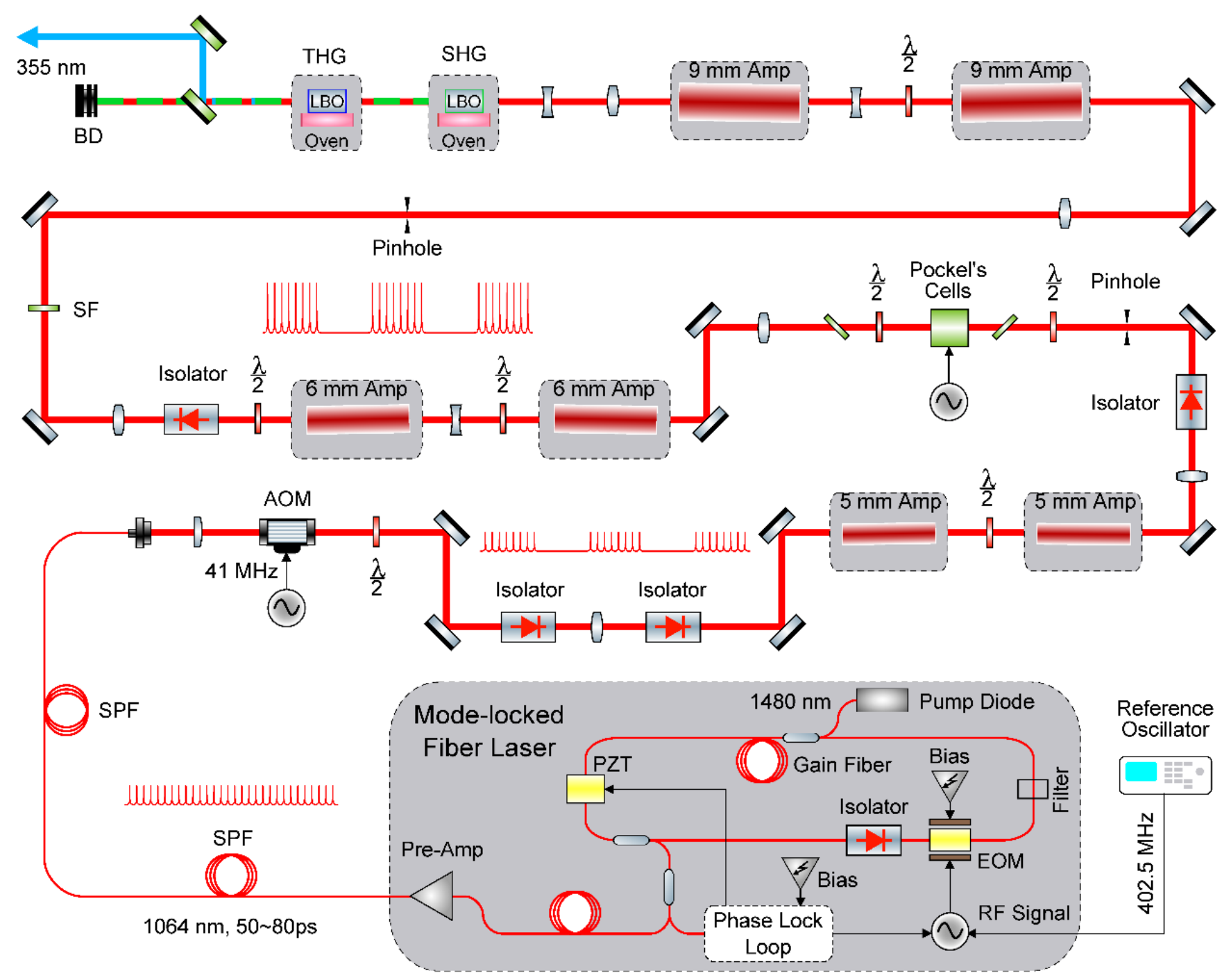

Figure 2 
(a)

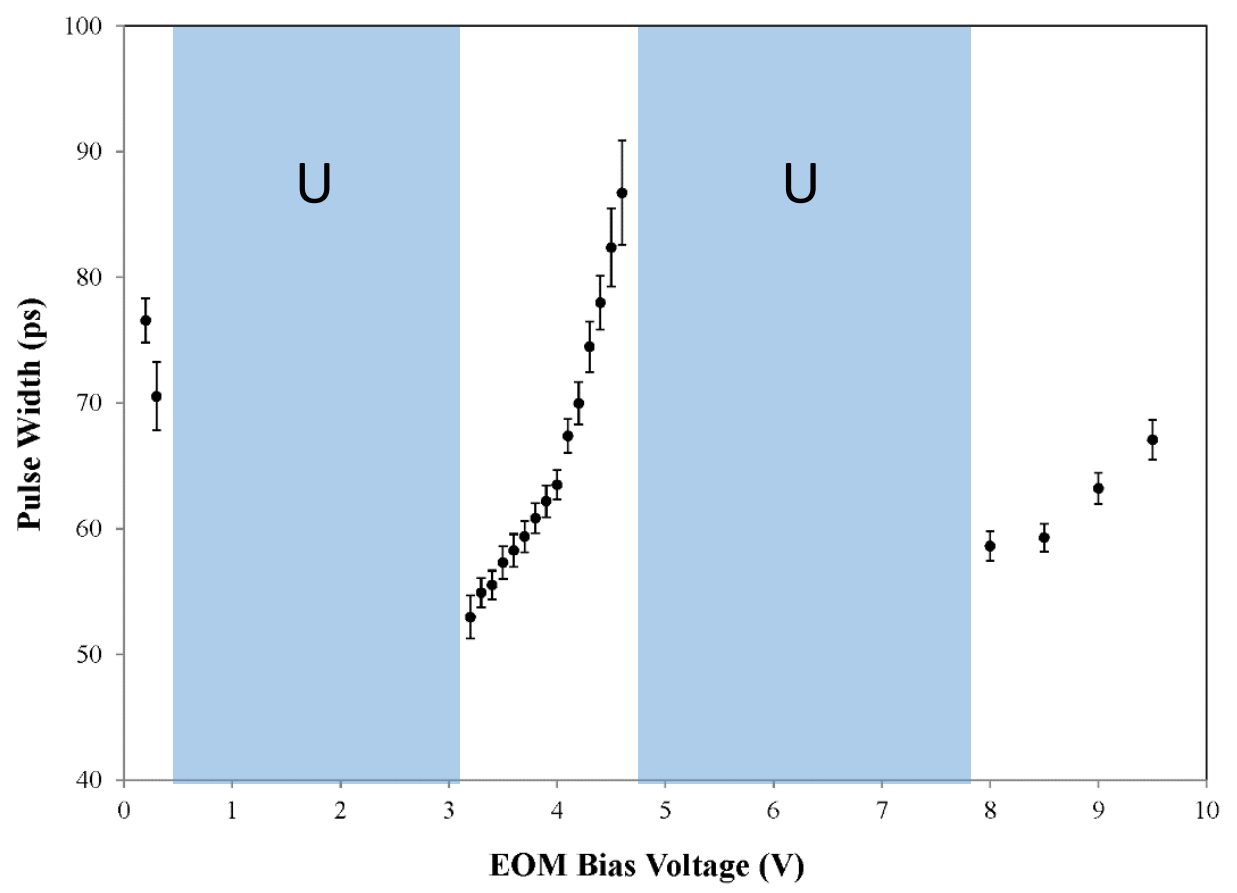

(b)

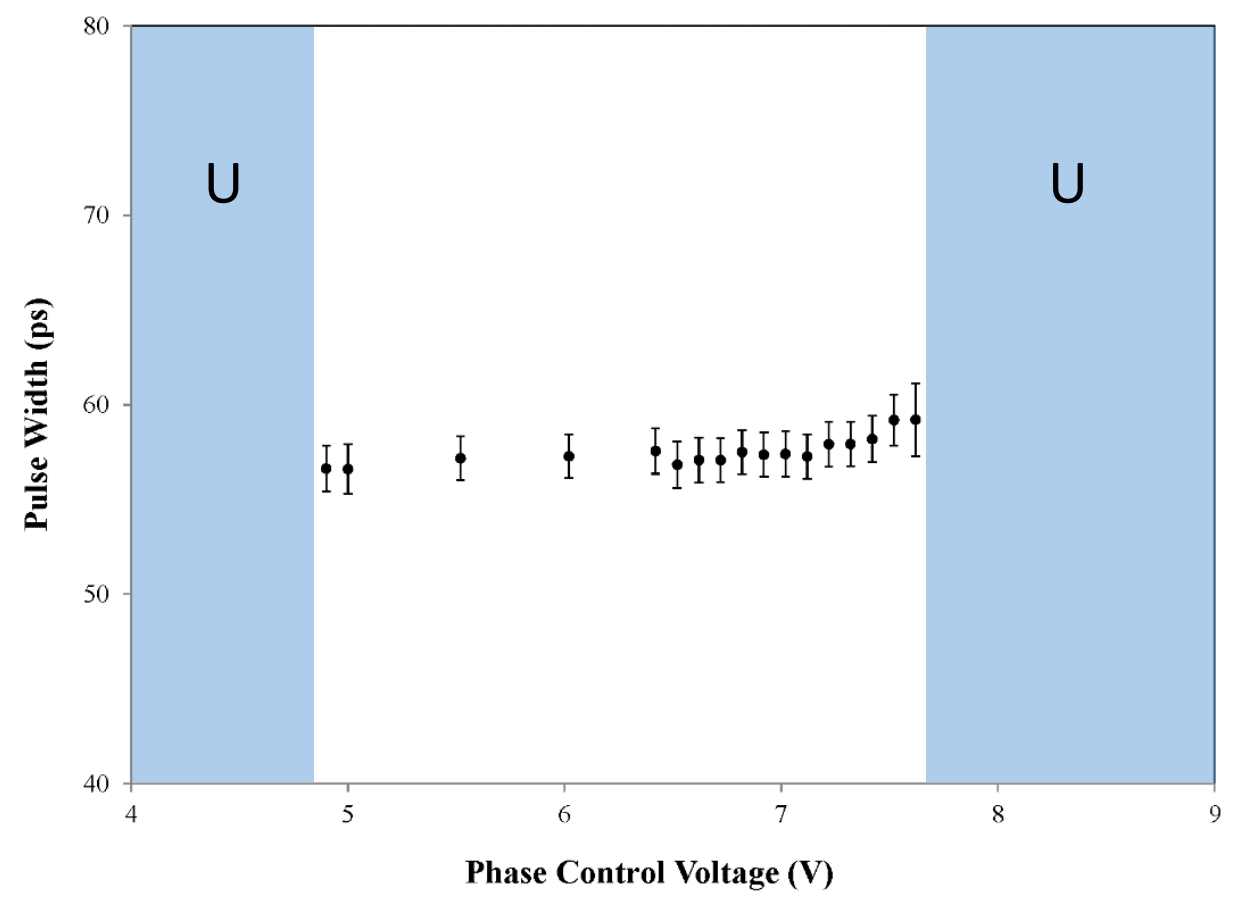

Figure 3 
(a)

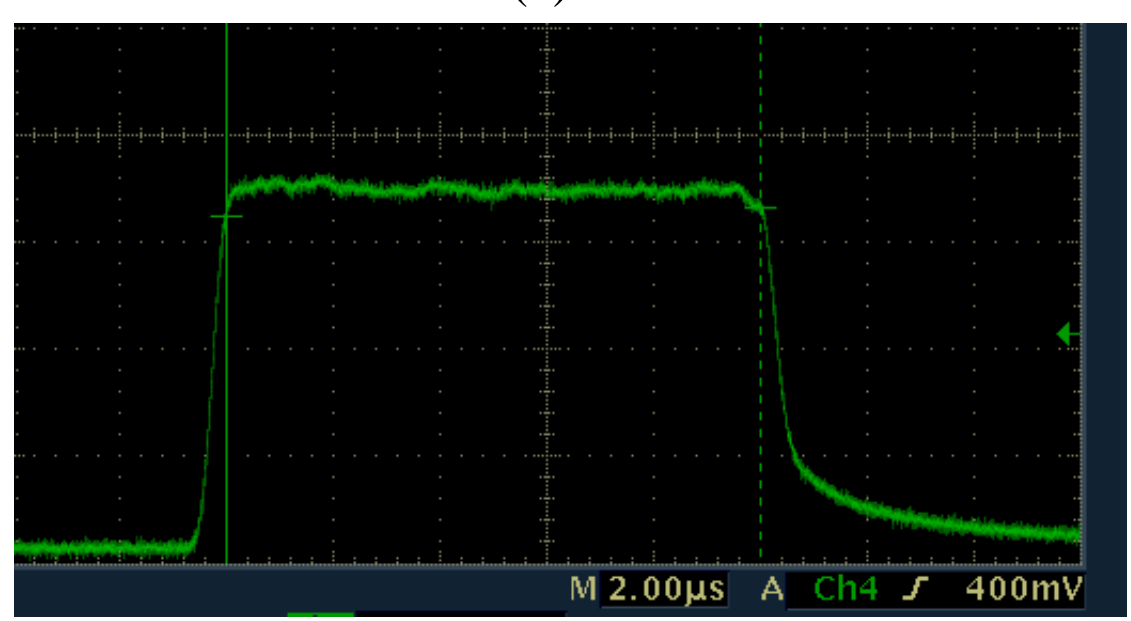

(b)

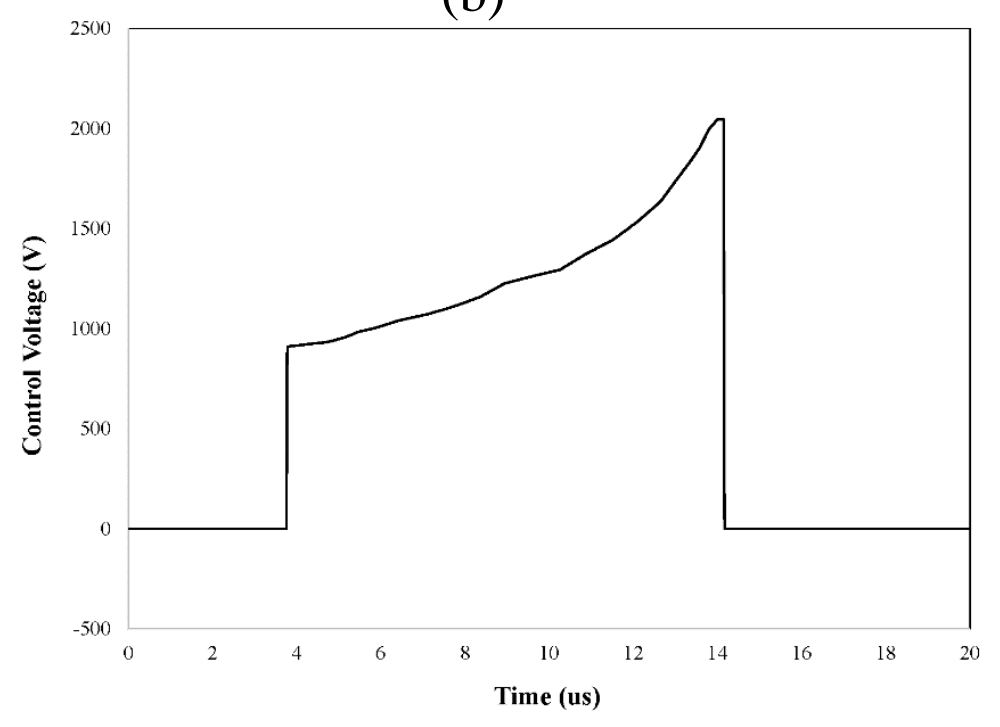

Figure 4 


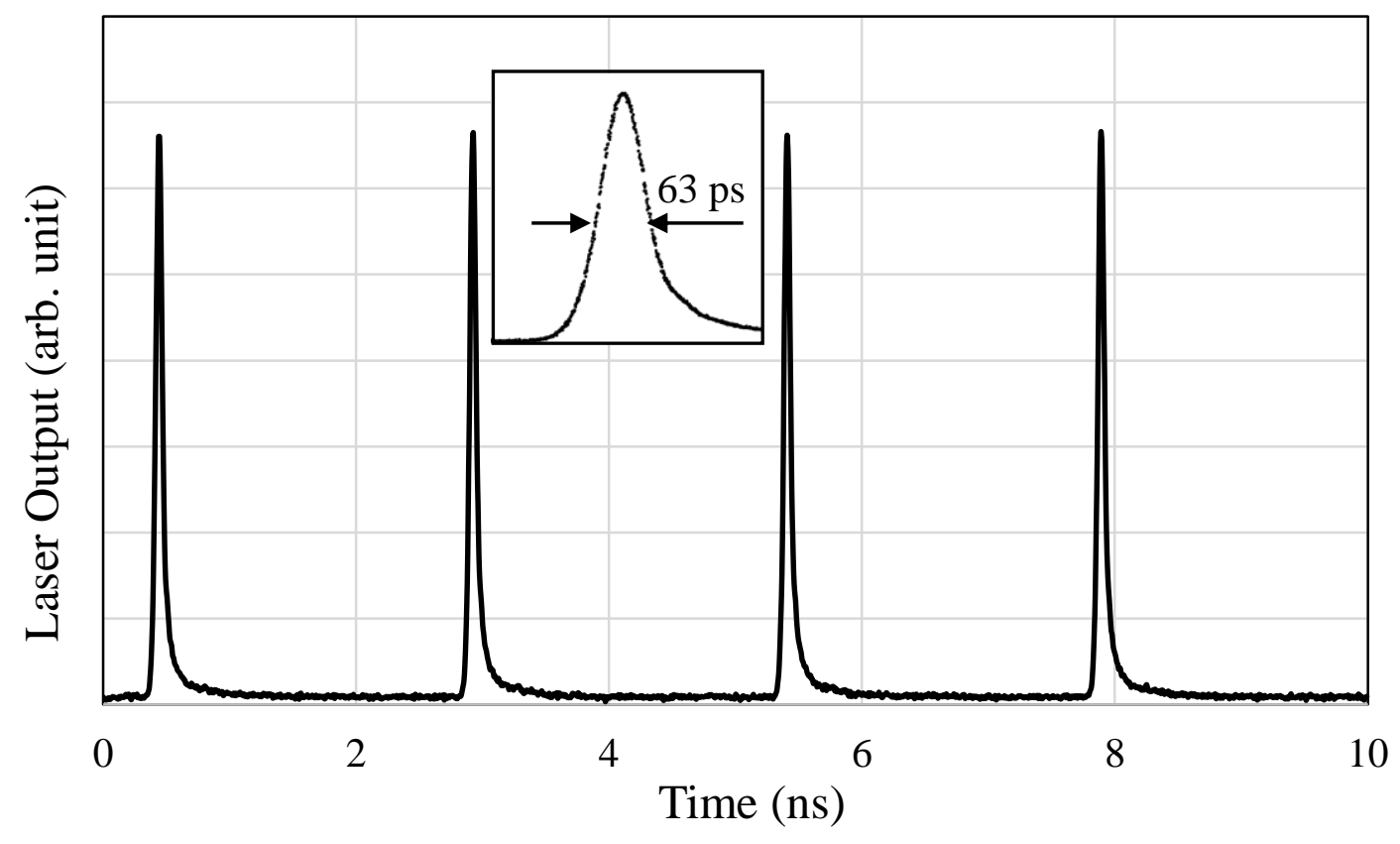

Figure 5 


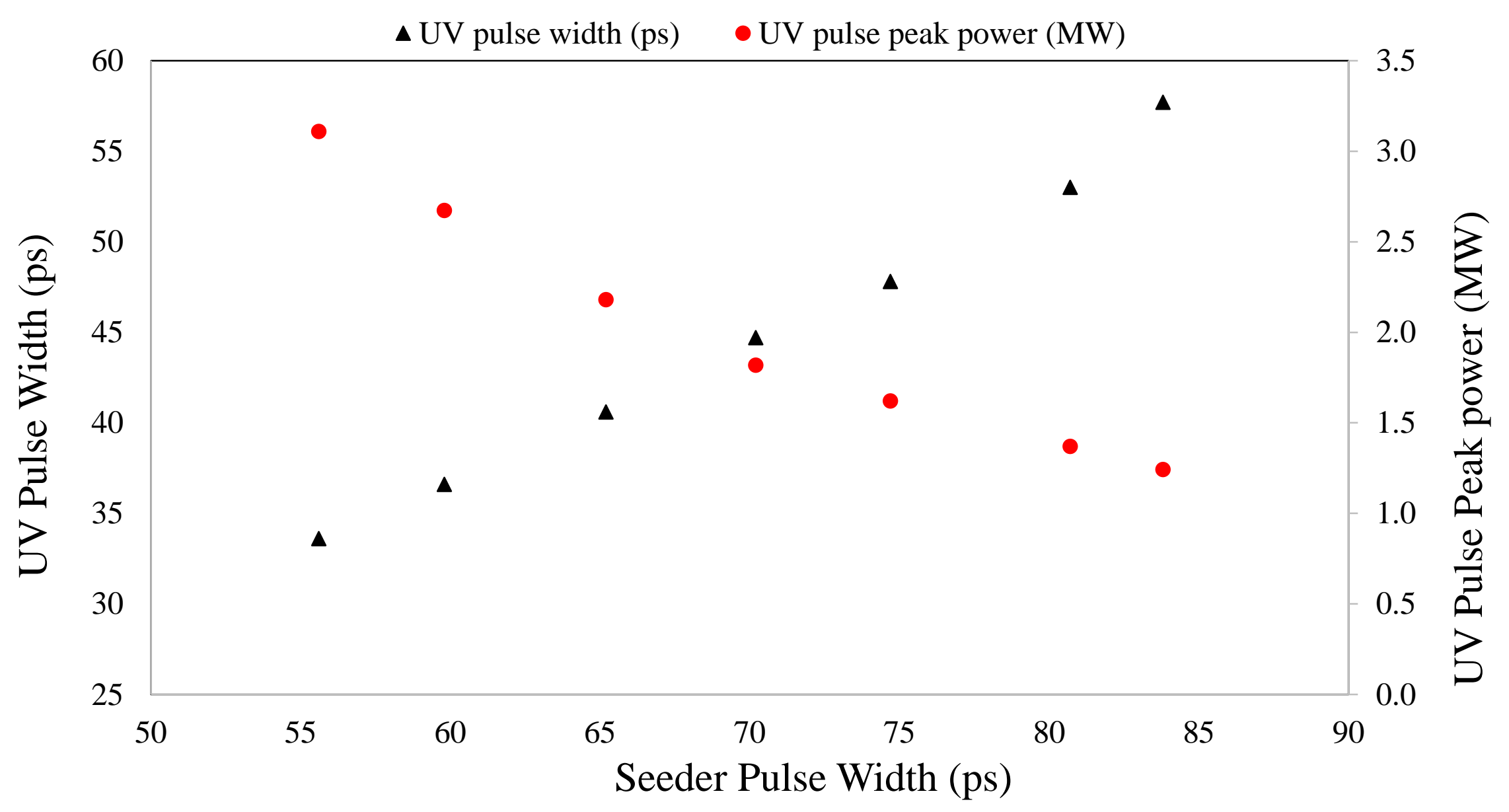

Figure 6 
(a)

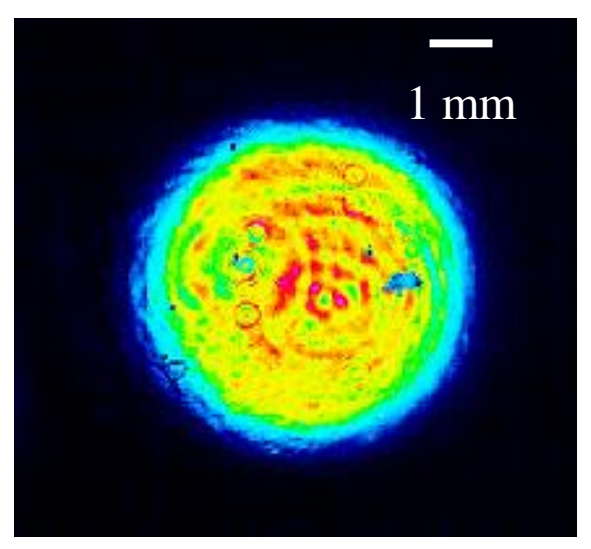

(b)

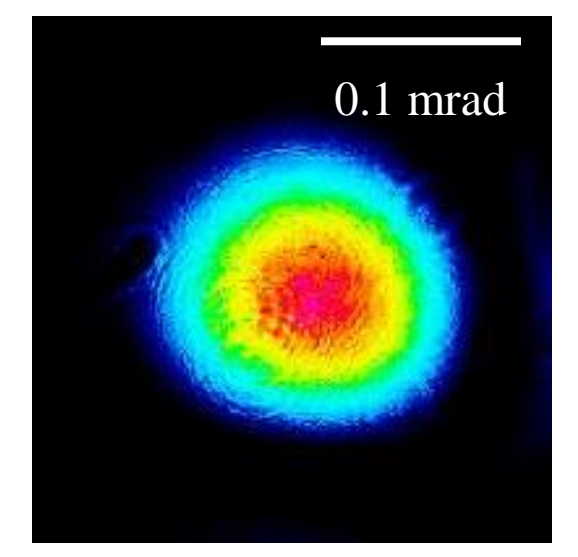

Figure 7 

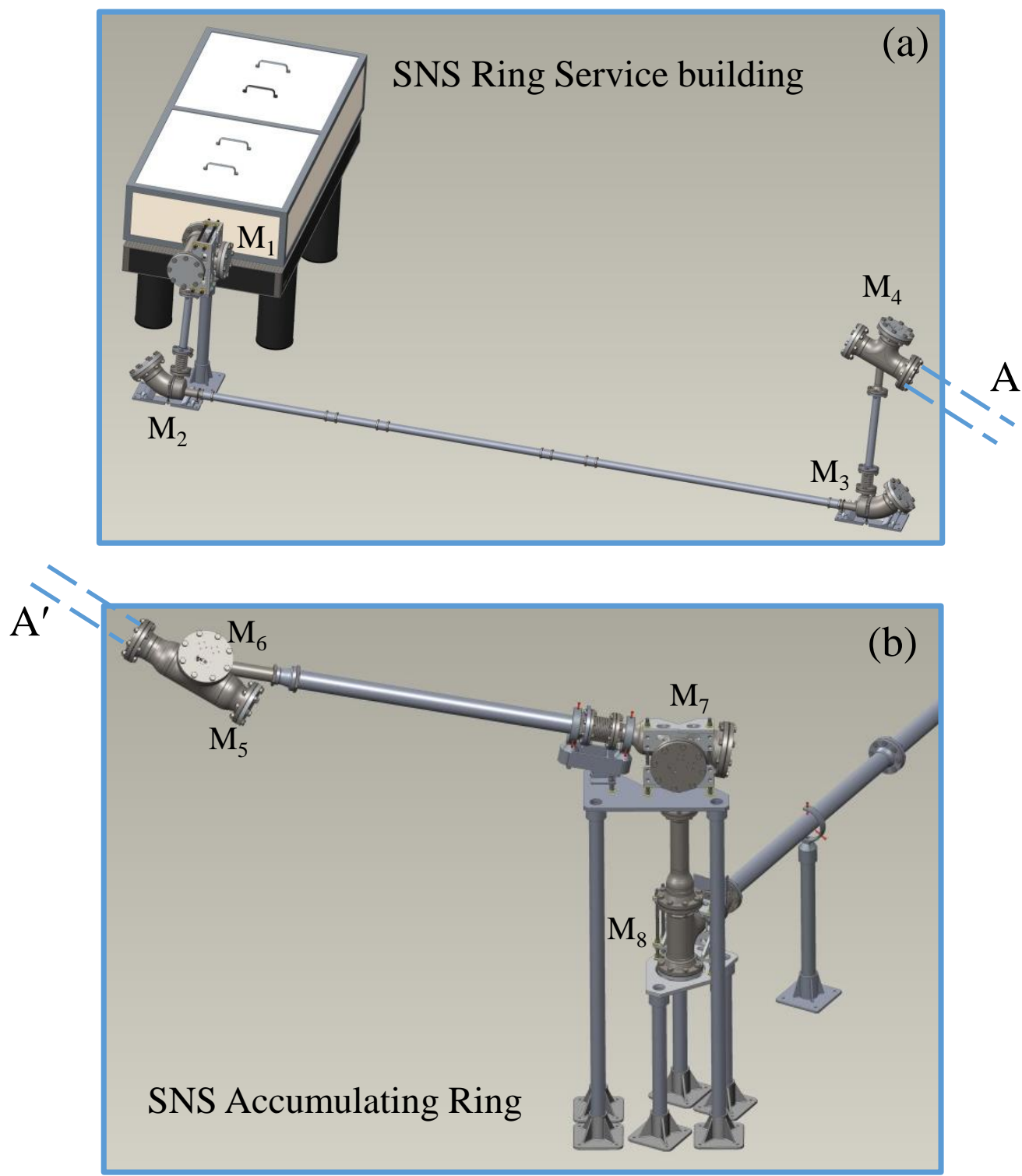

Figure 8 
(a)

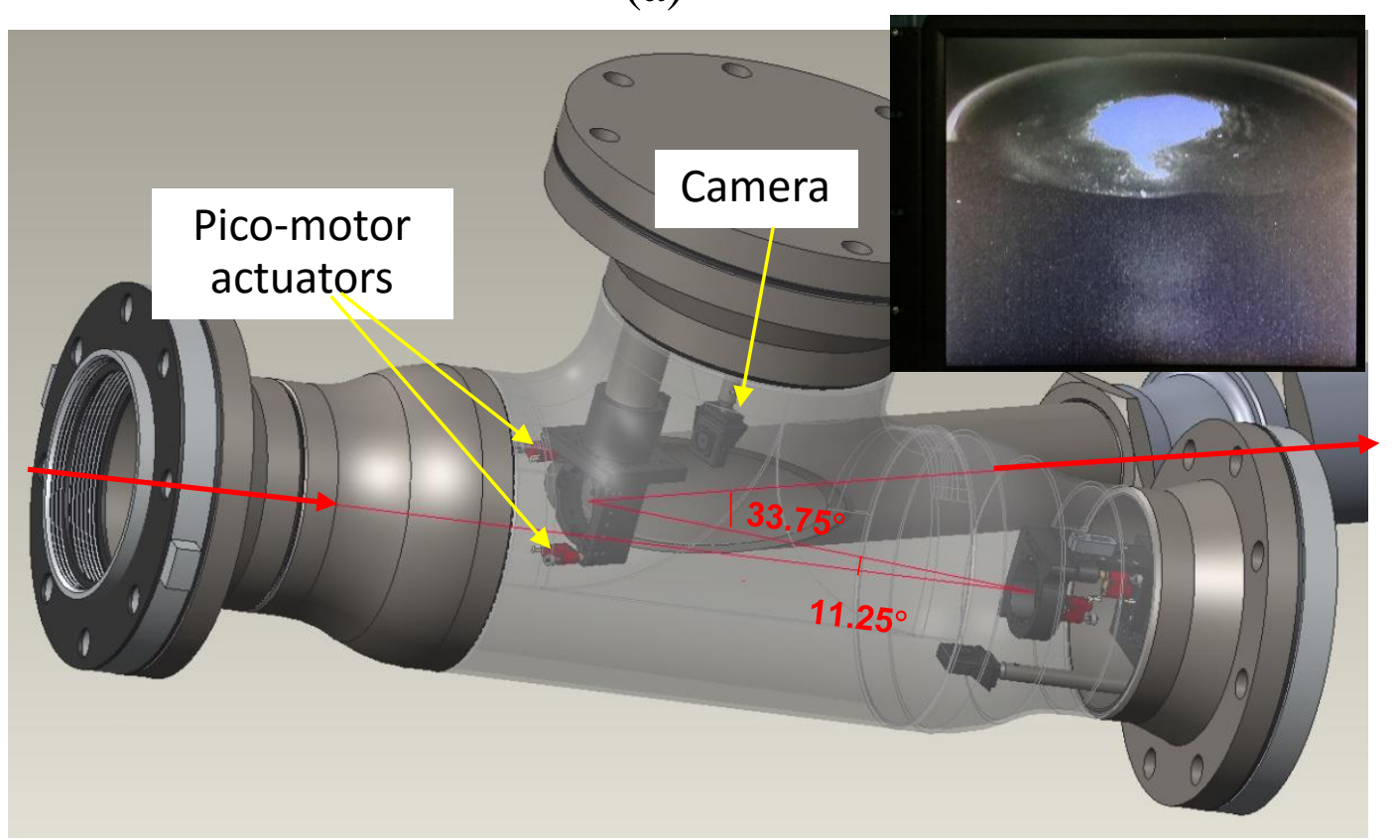

(b)

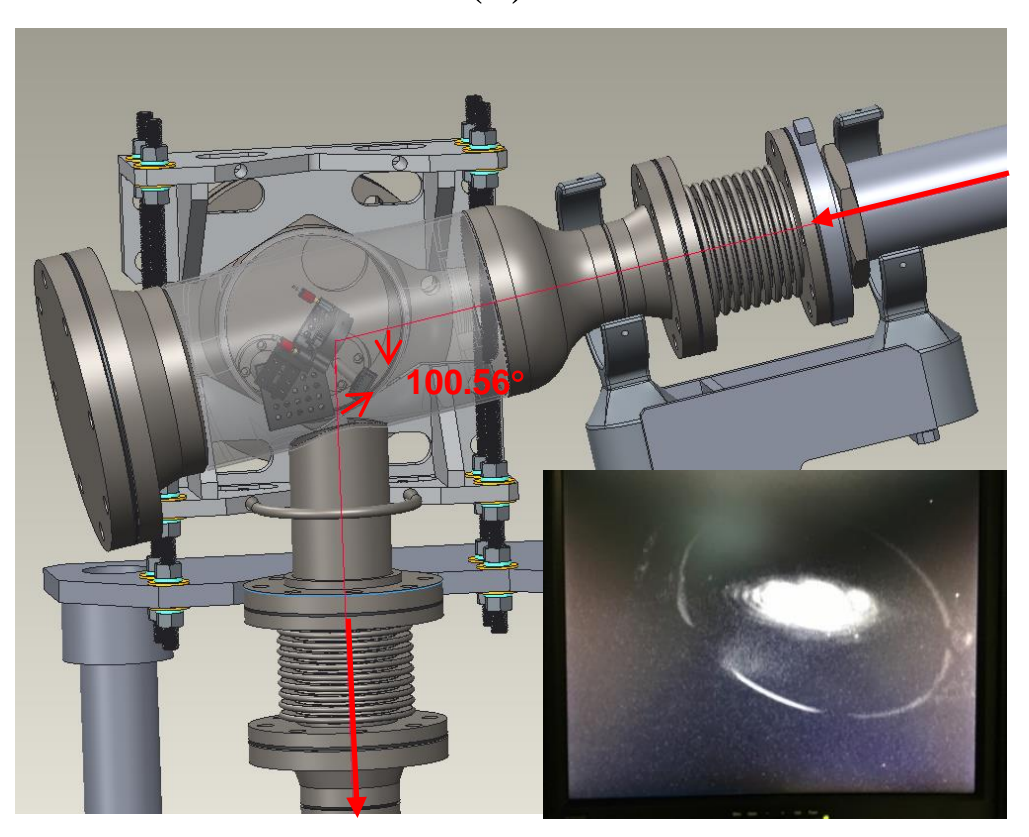

Figure 9 


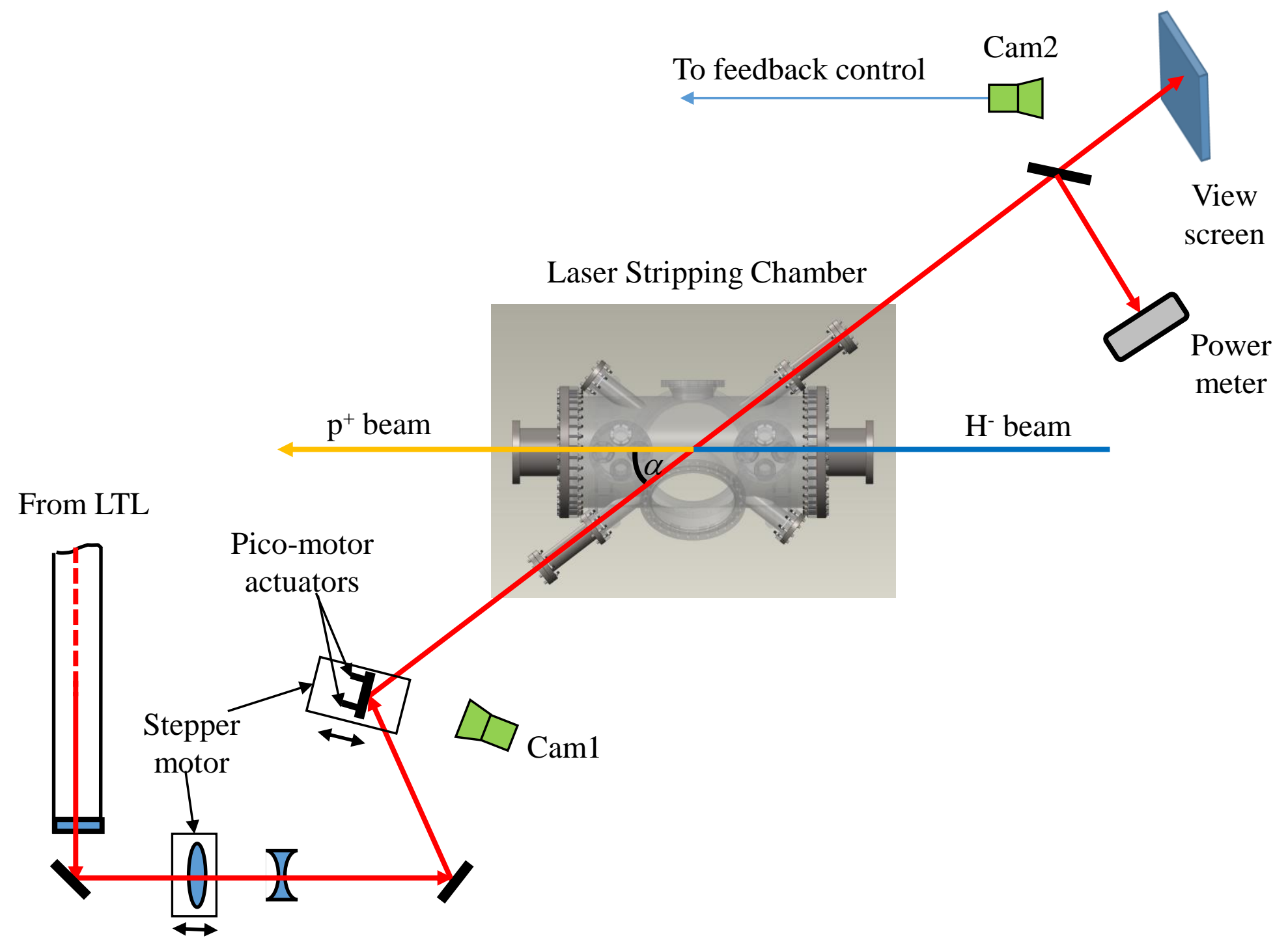

Figure 10 